\title{
Altered medial temporal activation related to local glutamate levels in subjects with prodromal signs of psychosis.
}

Valli, I ; Stone, J ; Mechelli, A ; Bhattacharyya, S ; Raffin, M ; Allen, P ; Fusar-Poli, P ; Lythgoe, D ; O'Gorman, R ; Seal, M ; McGuire, $\mathrm{P}$

\begin{abstract}
In individuals at high risk of psychosis, medial temporal dysfunction seemed related to a loss of the normal relationship with local glutamate levels. This study provides the first evidence that links medial temporal dysfunction with the central glutamate system in humans and is consistent with evidence that drugs that modulate glutamatergic transmission might be useful in the treatment of psychosis.
\end{abstract}

DOI: https://doi.org/10.1016/j.biopsych.2010.08.033

Posted at the Zurich Open Repository and Archive, University of Zurich

ZORA URL: https://doi.org/10.5167/uzh-56004

Journal Article

Accepted Version

Originally published at:

Valli, I; Stone, J; Mechelli, A; Bhattacharyya, S; Raffin, M; Allen, P; Fusar-Poli, P; Lythgoe, D; O'Gorman, R; Seal, M; McGuire, P (2011). Altered medial temporal activation related to local glutamate levels in subjects with prodromal signs of psychosis. Biological Psychiatry, 69(1):97-9.

DOI: https://doi.org/10.1016/j.biopsych.2010.08.033 


\section{Altered medial temporal activation related to local glutamate levels in subjects with prodromal signs of psychosis}

Isabel Valli ${ }^{\text {a }}$, James Stone ${ }^{\text {a, d }}$, Andrea Mechelli ${ }^{\text {a }}$, Sagnik Bhattacharyya ${ }^{\text {a }}$, Marie Raffin ${ }^{\text {a, e }}$, Paul Allen ${ }^{\text {a }}$, Paolo Fusar-Poli ${ }^{\mathrm{a}, \mathrm{f}}$, David Lythgoe ${ }^{\mathrm{b}}$, Ruth O'Gorman ${ }^{\mathrm{c}}$, Marc Seal ${ }^{\mathrm{g}}$, Philip McGuire ${ }^{\mathrm{a}}$

${ }^{a}$ Department of Psychosis Studies, King's College London, London, United Kingdom

${ }^{b}$ Department of Neuroimaging, King's College London, London, United Kingdom

${ }^{c}$ Institute of Psychiatry, King's College London, London, United Kingdom

${ }^{\mathrm{d}}$ Division of Experimental Medicine, Imperial College London, London, United Kingdom

${ }^{\mathrm{e}}$ Department of Child and Adolescent Psychiatry, Université Pierre et Marie Curie, Paris, France

${ }^{\mathrm{f}}$ Section of Psychiatry, Department of Behavioural Health Sciences, University of Pavia, Pavia, Italy

${ }^{\mathrm{g}}$ Developmental and Functional Brain Imaging Group, Murdoch Childrens Research Institute, Victoria, Australia

Key words: Psychosis, medial temporal cortex, glutamate, episodic memory, fMRI, MRS

Words abstract: 197

Words text: 1548

Figures: 1

Supplementary material: 1 text, 5 tables, 7 figures

Address for correspondence: Dr. Isabel Valli, Department of Psychosis Studies, PO67, Institute of Psychiatry, De Crespigny Park 16, SE58AF London UK.

Phone $+44(0) 7788482397$

Fax +44(0)2078480976

e-mail:isabel.valli@kcl.ac.uk 


\begin{abstract}
Background: Both medial temporal cortical dysfunction and perturbed glutamatergic neurotransmission are regarded as fundamental pathophysiological features of psychosis. However, while animal models of psychosis suggest that these two abnormalities are inter-related, their relationship in humans has yet to be investigated.
\end{abstract}

Methods: We used a combination of functional MRI (fMRI) and MR spectroscopy (MRS) to investigate the relationship between medial temporal activation during an episodic memory task and local glutamate levels in 22 individuals with an At Risk Mental State for psychosis and 14 healthy volunteers.

Results: We observed a significant between group difference in the coupling of medial temporal activation with local glutamate levels. In controls, medial temporal activation during episodic encoding was positively associated with medial temporal glutamate. However, in the clinical population, medial temporal activation was reduced and the relationship with glutamate was absent.

Conclusions: In individuals at high risk of psychosis medial temporal dysfunction appeared related to a loss of the normal relationship with local glutamate levels. This study provides the first evidence that links medial temporal dysfunction with the central glutamate system in humans, and is consistent with evidence that drugs that modulate glutamatergic transmission may be useful in the treatment of psychosis. 


\section{INTRODUCTION}

Psychotic disorders have frequently been associated with alterations in the structure and function of the medial temporal cortex $(1,2)$, and abnormalities in this region are thought to underlie the memory impairments that are evident in patients with psychosis at the behavioural level (3). An independent body of evidence indicates that perturbed glutamatergic neurotransmission is a key neurochemical feature of psychosis. N-methyl-D-aspartate glutamate (NMDA) receptor antagonists, such as ketamine, induce acute psychotic symptoms and impair memory performance (4), and psychotic disorders are associated with increased glutamine in the anterior cingulate cortex and thalamus (5), and a reduction in activated hippocampal NMDA receptor density (6) and NMDA receptor subunit mRNA (7). Animal models of psychosis and circuit analyses suggest that glutamatergic and medial temporal abnormalities are inter-related, with medial temporal cortex considered critical for the memory impairments observed after NMDA antagonists administration and in psychosis (8). The onset of psychotic disorders is preceded by a prodromal phase characterised by attenuated psychotic symptoms and a decline in global function. Recent studies in this phase of the disorder suggest that both hippocampal dysfunction and perturbed glutamate function are evident before the clinical expression of illness $(9,10)$, and that its onset is associated with progressive volumetric changes in the medial temporal cortex (11).

We examined the relationship between medial temporal function and central glutamate levels in people presenting with prodromal symptoms of psychosis. We first predicted, on the basis of previous studies $(10,12)$, that they would show altered medial temporal activation when performing a memory task, and alterations in regional glutamate levels. We then tested our main hypothesis, that the degree of medial temporal dysfunction would be related to the alteration in regional glutamate levels. 


\section{METHODS AND MATERIALS}

We used a combination of functional magnetic resonance imaging (fMRI) and proton magnetic resonance spectroscopy $\left({ }^{1} \mathrm{H}-\mathrm{MRS}\right)$ in the same individuals. The study was approved by the Joint South London and Maudsley and The Istitute of Psychiatry NHS research ethics committee, and all participants gave written informed consent to participate after a complete description of the study.

\section{Subjects}

Twenty-two subjects with an At Risk Mental State (ARMS) for psychosis (13), and a matching group of sixteen healthy volunteers took part in the study. However two of the control subjects were subsequently excluded, due to the poor quality of their MRS data in the hippocampal region. Participants were aged 18 to 30 years and excluded if their IQ was below 70, if there was a history of a neurological disorder or if they met DSM-IV criteria for a substance abuse disorder. The clinical population was recruited from Outreach and Support in South London (OASIS), a clinical service for people at high risk of developing a psychotic disorder, where subjects were assessed by two expert clinicians using the Comprehensive Assessment of At Risk Mental States (CAARMS, (13)) and diagnosis confirmed at a consensus clinical meeting. All subjects were antipsychotic naïve at the time of scanning, 3 subjects were receiving antidepressant treatment. The ARMS subjects were matched based on age, sex and IQ to a sample of healthy controls selected from the same sociodemographic area and recruited via advertisement (supplementary Table S1). All participants were right handed, as evaluated using the Lateral Preferences Inventory (14) and native English speakers.

\section{Clinical Measures}

Current symptoms were assessed in all the participants at the time of scanning using the CAARMS and the Positive and Negative Symptom Scale (PANSS, (15)). Premorbid IQ was measured with the Wide Range Achievement Test-Revised (WRAT-R, (16)). 


\section{Imaging}

${ }^{1} \mathrm{H}$-MRS was used to measure glutamate levels in the medial temporal cortex, anterior cingulate cortex, and thalamus (10). During the functional MRI session, subjects performed a verbal episodic memory paradigm that normally engages the medial temporal cortex (12) (supplementary Tables S3 and S4). During an encoding condition they read words aloud and were asked to remember them. In a recognition condition, they were presented with a subset of these words, mixed with novel, unstudied words, and asked whether they remembered them. To examine the correlation between BOLD response during the task and glutamatergic function, beta values were extracted from a region showing a between group difference in activation, and Pearson's correlation was performed in SPSS version 16.0 (SPSS inc. Chicago, Illinois, USA) to evaluate the association with local glutamate levels. Cook's distance test and leverage plot were used to assess the effect of potential outliers and influential cases (details of imaging procedures and data analysis in online supplementary Methods).

\section{RESULTS}

Consistent with previous studies (12), during verbal encoding the ARMS group showed reduced activation relative to controls in the left parahippocampal gyrus (PHG; $\mathrm{p}=0.047 \mathrm{FWE}$; fig 1), where the degree of activation in the ARMS group was directly correlated with task performance (the number of words correctly recalled during the subsequent recognition condition; $r=0.497, d f=20$, $\mathrm{p}=0.019)$. In controls, activation in this cluster during encoding was positively correlated with left medial temporal glutamate levels $(\mathrm{r}=0.592, \mathrm{df}=12, \mathrm{p}=0.026)($ Fig 1$)$, whereas there was a negative correlation in the ARMS subjects $(\mathrm{r}=-0.447, \mathrm{df}=20, \mathrm{p}=0.037)$. When Cook's distance test and leverage plot were used, the result in the ARMS group appeared driven by one case. When this subject was excluded the correlation in the ARMS group was no longer significant $(\mathrm{r}=-0.318$, $\mathrm{df}=19, \mathrm{p}=0.16$ ) (fig 1). To assess the potential effects of antidepressant treatment on the data, we 
al.

performed a sensitivity analysis, repeating the correlation after the exclusion of the three medicated ARMS subjects. This did not alter the results: again, there was no significant relationship between activation in the left parahippocampal cluster and medial temporal glutamate $(\mathrm{r}=-0.414, \mathrm{df}=14$, $\mathrm{p}=0.088$ ). Fisher $\mathrm{r}$ to $\mathrm{z}$ transformation was used to formally assess the difference in the correlations within each group, and showed a significant difference between the respective correlation coefficients $(Z=2.64 ; \mathrm{p}<0.01)$.

There were no significant group differences in regional glutamate levels (although there was a trend $[p=0.079]$ for a reduction in the thalamus in the ARMS group; details in Table S5 of online supplementary material). There were no group differences in medial temporal activation during the recognition (as opposed to the encoding) condition. We therefore did not assess the relationship between glutamate levels and medial temporal activation during the recognition phase of the task.

***FIGURE 1 ABOUT HERE***

Fig 1: Left parahippocampal (PHG) region $(x=-18 y=-30 \mathrm{z}=-20)$ where ARMS subjects showed less activation (in arbitrary units) than controls during encoding ( $\mathrm{p}=0.047 \mathrm{FWE}$ ). In this region, activation in controls was positively correlated with left medial temporal glutamate levels, but there was no correlation in the ARMS group.

\section{DISCUSSION}

These results suggest that medial temporal dysfunction in people with prodromal symptoms of psychosis is related to a loss of the normal relationship between function in this region and local glutamate levels. While both medial temporal dysfunction and altered glutamate levels have each been described separately in relation to psychosis in humans $(2,5)$, this is the first time a link 
al.

between them has been demonstrated in the same subjects. A direct relationship between them provides support to contemporary animal models of psychosis, which propose that medial temporal dysfunction is associated with a disturbance of glutamate neurotransmission (8).

NMDA receptor antagonists can induce neuronal damage in the limbic cortex of rodents. This effect is prevented by pretreatment with gamma-aminobutyric acid (GABA) receptor agonists, suggesting that it involves blockade of excitatory glutamate receptors on GABAergic inhibitory interneurons (17). An effect on GABAergic interneurons may also underlie the emergence of psychotic symptoms observed following NMDA receptor blockade in humans (18), and a similar mechanism has been hypothesised for the glutamate model of psychosis (18). The specificity of our findings to the encoding, as opposed to the recognition phase of the memory task is consistent with evidence that infusion of NMDA receptor antagonists in the limbic cortex of rodents impairs memory encoding, but not recognition (19). A similar impairment has been observed in humans, where ketamine administration induces robust episodic memory impairments affecting mainly early consolidation processes (20) during the encoding of information (21). This may reflect the role of hippocampal NMDA receptors in the induction of the activity-dependent synaptic plasticity that underlies memory (22).

While our results suggest that medial temporal dysfunction in people at high risk of psychosis is related to regional glutamate levels, they cannot reveal the direction of causality, nor whether the MRS measure is related to activity in medial temporal pyramidal neurons, in the terminals of afferent projections from other regions, or abnormalities at a receptor level.

A further caveat is that it is difficult to conclusively discriminate between the signals corresponding to glutamate and glutamine with the MRS method we used. Due to the partial overlap of glutamate and glutamine resonances at 3T, glutamate levels in the present study may thus include a contribution from glutamine. Simulation studies suggest that glutamine 
al.

may account for $10-15 \%$ of the glutamate concentration at $3 \mathrm{~T}$ (23). The group sizes in the present study were modest and unequal, and replication of the findings in a larger sample is indicated. However, although we cannot exclude the effect of sample size on power, this is unlikely to have affected the main findings of the present study. The positive correlation observed between parahippocampal activation and hippocampal glutamate levels was present in the control group with a smaller sample size, but absent in the ARMS group with a larger sample. In conclusion, the findings are consistent with evidence that drugs which modulate glutamate transmission may be useful in patients with psychosis (24). Moreover, because the changes we observed predated the clinical expression of psychosis, they suggest that glutamatergic drugs may be able to influence the risk of developing the first episode of psychosis.

This research was supported by Medical Research Council Clinical Training Fellowships awarded to IV (Grant No G0601437), JS (Grant No. G0500477) and SB (Grant No G0501755) as well as a National Alliance for Research on Schizophrenia and Depression (NARSAD) Young Investigator Award (PA).

We thank Mary McLean and Gareth Barker for very useful discussion, and also all the participants and the members of the Outreach and Support in South London (OASIS) team.

The authors report no biomedical financial interests or potential conflicts of interest. 
References

1. Vita A, De Peri L, Silenzi C, Dieci M (2006): Brain morphology in first-episode schizophrenia: a meta-analysis of quantitative magnetic resonance imaging studies. Schizophr Res. 82:75-88.

2. Achim AM, Lepage M (2005): Episodic memory-related activation in schizophrenia: metaanalysis. Br J Psychiatry. 187:500-509.

3. Boyer P, Phillips JL, Rousseau FL, Ilivitsky S (2007): Hippocampal abnormalities and memory deficits: new evidence of a strong pathophysiological link in schizophrenia. Brain Res Rev. 54:92-112.

4. Newcomer JW, Farber NB, Jevtovic-Todorovic V, Selke G, Melson AK, Hershey T, et al. (1999): Ketamine-induced NMDA receptor hypofunction as a model of memory impairment and psychosis. Neuropsychopharmacology. 20:106-118.

5. Theberge J, Bartha R, Drost DJ, Menon RS, Malla A, Takhar J, et al. (2002): Glutamate and glutamine measured with 4.0 T proton MRS in never-treated patients with schizophrenia and healthy volunteers. Am J Psychiatry. 159:1944-1946.

6. Pilowsky LS, Bressan RA, Stone JM, Erlandsson K, Mulligan RS, Krystal JH, et al. (2006): First in vivo evidence of an NMDA receptor deficit in medication-free schizophrenic patients. Mol Psychiatry. 11:118-119.

7. Gao XM, Sakai K, Roberts RC, Conley RR, Dean B, Tamminga CA (2000): Ionotropic glutamate receptors and expression of N-methyl-D-aspartate receptor subunits in subregions of human hippocampus: effects of schizophrenia. Am J Psychiatry. 157:1141-1149.

8. Lisman JE, Coyle JT, Green RW, Javitt DC, Benes FM, Heckers S, et al. (2008): Circuitbased framework for understanding neurotransmitter and risk gene interactions in schizophrenia. Trends Neurosci. 31:234-242.

9. Hurlemann R, Jessen F, Wagner M, Frommann I, Ruhrmann S, Brockhaus A, et al. (2008): Interrelated neuropsychological and anatomical evidence of hippocampal pathology in the at-risk mental state. Psychol Med. 38:843-851.

10. Stone JM, Day F, Tsagaraki H, Valli I, McLean MA, Lythgoe DJ, et al. (2009): Glutamate dysfunction in people with prodromal symptoms of psychosis: relationship to gray matter volume. Biol Psychiatry. 66:533-539.

11. Pantelis C, Velakoulis D, McGorry PD, Wood SJ, Suckling J, Phillips LJ, et al. (2003): Neuroanatomical abnormalities before and after onset of psychosis: a cross-sectional and longitudinal MRI comparison. Lancet. 361:281-288.

12. Allen P, Seal ML, Valli I, Fusar-Poli P, Perlini C, Day F, et al. (2009): Altered Prefrontal and Hippocampal Function During Verbal Encoding and Recognition in People With Prodromal Symptoms of Psychosis. Schizophr Bull.

13. Yung AR, Phillips LJ, McGorry PD, McFarlane CA, Francey S, Harrigan S, et al. (1998): Prediction of psychosis. A step towards indicated prevention of schizophrenia. Br J Psychiatry Suppl. 172:14-20.

14. Coren S (1993): Measurement of handedness via self-report: the relationship between brief and extended inventories. Percept Mot Skills. 76:1035-1042.

15. Kay SR, Fiszbein A, Opler LA (1987): The positive and negative syndrome scale (PANSS) for schizophrenia. Schizophr Bull. 13:261-276.

16. Jastak S WS (1984): The Wide Range Achievement Test: Revised Administration Manual. Wilmington: Jastak Associates. 
al.

17. Olney JW, Farber NB (1995): Glutamate receptor dysfunction and schizophrenia. Arch Gen Psychiatry. 52:998-1007.

18. Sharp FR, Tomitaka M, Bernaudin M, Tomitaka S (2001): Psychosis: pathological activation of limbic thalamocortical circuits by psychomimetics and schizophrenia? Trends Neurosci. 24:330-334.

19. Day M, Langston R, Morris RG (2003): Glutamate-receptor-mediated encoding and retrieval of paired-associate learning. Nature. 424:205-209.

20. Parwani A, Weiler MA, Blaxton TA, Warfel D, Hardin M, Frey K, et al. (2005): The effects of a subanesthetic dose of ketamine on verbal memory in normal volunteers. Psychopharmacology (Berl). 183:265-274.

21. Morgan CJ, Curran HV (2006): Acute and chronic effects of ketamine upon human memory: a review. Psychopharmacology (Berl). 188:408-424.

22. Bliss TV, Collingridge GL (1993): A synaptic model of memory: long-term potentiation in the hippocampus. Nature. 361:31-39.

23. Hancu I (2009): Optimized glutamate detection at 3T. J Magn Reson Imaging. 30:11551162.

24. Patil ST, Zhang L, Martenyi F, Lowe SL, Jackson KA, Andreev BV, et al. (2007):

Activation of $\mathrm{mGlu} 2 / 3$ receptors as a new approach to treat schizophrenia: a randomized Phase 2 clinical trial. Nat Med. 13:1102-1107. 